\title{
Stability Indicating LC-MS Method Development and Validation for the Study of the Forced Degradation Behavior of Pimobendan Drug
}

\author{
Atchuta Ramakrishna Gudapati ${ }^{1}, *$, Srinivasarao Pakalapati ${ }^{1}$, Chidananda Swamy Rumalla ${ }^{1}$, Benarji Patrudu Tammina ${ }^{2}$ \\ 'Department of Medicinal Chemistry, GVK Biosciences Pvt. Ltd, IDA Nacharam, Hyderabad,Telangana, INDIA. \\ 2Department of Chemistry, Gitam School of Science, Rudraram, Patancheru Mandal, Hyderabad, Telangana, INDIA.
}

\begin{abstract}
Objectives: Analytical methods are necessary for the field of pharmaceuticals to discover the drug, determination of drugs and metabolites in biological matrices. The present study aimed to develop liquid chromatography-tandem mass spectrometry (LC-MS) and validation for quantification of Pimobendan in the formulation. Methods: The mobile phase composition was methanol, acetonitrile and $0.1 \mathrm{M}$ phosphate buffers in the ratio of 45:15:40 (v/v) mixtures with isocratic elution. Prontosil ODS $\mathrm{C}_{18}$ column $(250 \times 4.6 \mathrm{~mm}, 5 \mu)$ was selected for separation with $264 \mathrm{~nm}$ of LC UV detector wavelength. Ionization mass spectrophotometer was analyzed at nitrogen gas flow at 300 Psi and $300^{\circ} \mathrm{C}$ of source temperature. Mass spectrometry analysis of Pimobendan drug fragments was performed by introducing column eluting into equipment for Q1 and MS scan. Results: The drug was eluted at $3.56 \mathrm{~min}$ retention and the calibration curve was achieved at $0.5-250 \mu \mathrm{g} / \mathrm{ml}$ standard concentration. Different parameters of validation (linearity, specificity, precision, accuracy, selectivity and robustness) were performed and results confirm that all the parameters are in the acceptable limit. In the
\end{abstract}

electrospray ionization spectra of Pimobendan $[\mathrm{M}+\mathrm{H}]$, positive ions were observed at an $\mathrm{m} / \mathrm{z}$ value of 335.42 . The product-ions of 335.42 were observed at $\mathrm{m} / \mathrm{z}$ value of $319.18,287.19,277.09,261.08,250.16,223.30$, 111.47 and 86.05. Conclusion: The proposed method is successfully achieved the acceptance criteria of all validation parameters and stability studies.

Key words: Pimobendan, Liquid Chromatography-Mass Spectrometry (LC-MS), Method Validation, Degradation compounds, Stability study, Quantification.

\section{Correspondence}

Mr. Atchuta Ramakrishna Gudapati

Department of Chemistry, Gitam School of Science, Rudraram, Patancheru Mandal, Hyderabad-502329, Telangana, INDIA.

Phone no: +91-9866092754

Email: atchutaramakrishnagudapati@gmail.com

DOI: : 10.5330/ijpi.2020.4.91

\section{INTRODUCTION}

Pimobendan (PBD) is phenyl benzimidazoles class veterinary medication used for the treatment of heart failure (Congestive heart failure) ${ }^{1,2}$ myxomatous mitral valve disease and dilated cardiomyopathy ${ }^{3,4}$ in dogs. It is frequently prescribed in combination with Angiotensinconverting-enzyme (ACE) inhibitors like benazepril/enalapril. ${ }^{5}$ The bit of leeway related with pimobendan is that it applies positive inotropic impacts without causing an expansion in myocardial oxygen demand. ${ }^{6}$ The chemical formula of the PBD is $\mathrm{C}_{19} \mathrm{H}_{18} \mathrm{~N}_{4} \mathrm{O}_{2}$. The chemical structure of the PBD is presented in Figure 1. It contains a benzimidazole moiety, where its imidazole ring is attached to a phenyl group. It is a calcium sensitizer and expands the coupling proficiency of heart troponin in the myofibril to the calcium particles that present in the systole. Side effects with the drug include loss of appetite, lethargy, diarrhea, dyspnea, azotemia and ataxia. ${ }^{7,8}$

Chromatography methods became an essential choice for the separation of analytes and stability-indicating studies of the drugs in the pharmaceutical industry. Stability studies of the drug involve various stress conduction like hydrolysis, oxidation and thermal degradation, etc. The stability studies require special attention in order to study the degradation products of the drug that exceeds the thresholds for identification and quantification found in stability studies. The spectra information of the results of drug stability-indicating methods is useful for the rapid identification of degradation products and useful for the structure clarification. A literature review of PBD analysis reveals that there are only two RP-HPLC methods ${ }^{9,10}$ were reported. LC-MS is an analytical technique, using LC as a separation system and MS as a detection system. ${ }^{10}$ LC-MS has a broad scope of use in different divisions, including biotechnology, food technology, environmental, pharmaceutical, cosmetic and agrochemical industries. ${ }^{11-13}$ Hence the present approach for the development and validation of the LC-MS method for quantitative and qualitative analysis of PBD would provide more detailed and accurate information of the drug during the analysis.

\section{MATERIALS AND METHODS}

\section{Instrumentation}

Chromatographic separation was achieved with Agilent make HPLC 1100 series with Quaternary pump (G1311), colcom G1316A thermostat column temperature control (COLCOM G1316A), Thermostatic auto sample (G 1329A) with volume capacity (0.1-1500 $\mu \mathrm{l})$ and Ultraviolet detector (G 1314A). Agilent Chem Station software was used for the operation and integration of the instrument. The HPLC system was coupled with Waters ZQ Mass Detector (model LAA 1369) with Waters Mass LYNX V4 software. The mass spectra obtained in ESI positive mode (ESI Turbo ion spray) with a mass range of 40-1000 Amu and quadruple analyzer with MS tune of $3.0 \mathrm{kV}$ (Capillary, $40 \mathrm{~V}$ cone, $3 \mathrm{~V}$ extractor and source temperature at $300^{\circ} \mathrm{C}$ used for analysis. 


\section{Preparation of solutions}

pH 6.4 phosphate buffer solution: About $8.395 \mathrm{~g}$ of Disodium hydrogen orthophosphate $\left(\mathrm{Na}_{2} \mathrm{HPO}_{4}\right)$ and $9.477 \mathrm{~g}$ of sodium dihydrogen phosphate $\left(\mathrm{NaH}_{2} \mathrm{PO}_{4}\right)$ was dissolved in one liter of Milli-Q water and then the $\mathrm{pH}$ was adjusted to 6.4 using $0.1 \mathrm{~N}$ hydrochloric acid solution.

Standard drug solution: An accurately weighed $100 \mathrm{mg}$ of standard drug PBD was taken into $100 \mathrm{ml}$ volumetric flask and dissolved with methanol to obtain the concentration of $1000 \mu \mathrm{g} / \mathrm{mL}$ of standard PBD drug solution. The concentrations required for the concentration of the calibration curve was prepared separately by proper dilutions. Equal volumes of selected concentrations of PBD were mixed separately and the mixture solution was used for the method development and validation studies.

\section{Method development}

The standard drug solution containing PBD at a concentration of $100 \mu \mathrm{g} /$ $\mathrm{mL}$ is initially used for method development studies. The maximum absorption wavelength $\left(\lambda_{\max }\right)$ of PBD was confirmed by scanning the standard drug in the wavelength range $200-400 \mathrm{~nm}$ in a UV spectrophotometer. System suitability, resolution, peak symmetry and responses factor are the key factors that are kept in consideration for optimization of the mobile phase. The mobile phase was confirmed by the change in different solvents in different ratios, the strength of organic modifier and different strengths of $\mathrm{pH}$ modifier. The separation was performed on different column configurations $\left(\mathrm{C}_{8}\right.$ and $\left.\mathrm{C}_{18}\right)$ and manufactures. The flow rate of the mobile phase was also modified to obtain a better resolution of the chromatogram. The chromatography conditions that achieve the best resolution, response and peak symmetry and considered as optimum conditions for further validation studies.

\section{Method Validation}

The method validation was conducted as per the guidelines given by $\mathrm{ICH},{ }^{14-16}$ including selectivity, sensitivity, accuracy, recovery, precision and linearity of the stability of PBD in different stress conditions. The selectivity and sensitivity of the method were verified by analyzing blank samples without the drug.

\section{System suitability}

The drug standard solution at a concentration of $100 \mu \mathrm{g} / \mathrm{mL}$ of PBD was analyzed six times in the developed method. System suitability was evaluated by the method of chromatography parameters such as retention time, theoretical plate number and asymmetric factor of the results were used and the number of theoretical plates of $>2000$ and tail factor $<2$ was considered as acceptable.

Linearity and range: Six different dilutions of PBD at a range of $0.5-250$ $\mu \mathrm{g} / \mathrm{mL}$ concentration were prepared and used for the evaluation of the linear calibration curve. The resultant peak area and the concentration of $\mathrm{PBD}$ prepared were used for linear least square regression analysis.

Precision and Ruggedness: Six replicate analysis of PBD standard solution containing $100 \mu \mathrm{g} / \mathrm{mL}$ was analyzed on the same day for intraday precision, six times in three days for interday precision and by the change in analyst for ruggedness study. The RSD (Relative standard deviation) of peak area response of PBD was calculated and a \% RSD of $<2$ in each study for both the drug was considered as the method is precise and rugged.

Robustness: The Robustness study was carried out by the slight variation in optimized method conditions developed for PBD, such as change in \pm $5 \mathrm{~nm}$ of detector wavelength, $\pm 5 \mathrm{~mL}$ variation in mobile phase organic and $\mathrm{pH}$ modifier and \pm 0.1 factor of mobile phase $\mathrm{pH}$. In each condition, the standard solution containing $100 \mu \mathrm{g} / \mathrm{ml}$ of PBD was analyzed and the percentage of change in peak area was calculated and the $\%$ change of less than 2 was considered as the method is robust.

Recovery: In the recovery study, 50\%, 100\% and 150\% level concentrations were spiked to a known concentration of $100 \mu \mathrm{g} / \mathrm{mL}$ of PBD. The PBD spiked solutions were analyzed and compared with standard calibration results and the percentage of recovery of PBD with the proposed method was calculated and the \% recovery of more than 98 and less than 102 was found to be the method is accurate.

Sensitivity: The detection limit (LOD) and quantification limit (LOQ) of PBD for proposed method conditions confirm the sensitivity of the method. The Signal/noise $(\mathrm{S} / \mathrm{N})$ ratio of chromatographic detection of PBD to the noise observed for the blank solution was used for the determination of the sensitivity of the method.

\section{Forced degradation studies}

The acidic, alkaline and peroxide (oxidative degradation) degradation studies were carried out by $50 \mathrm{mg}$ of standard drug PBD was mixed with $50 \mathrm{~mL}$ of $0.1 \mathrm{~N} \mathrm{HCl}, 0.1 \mathrm{~N} \mathrm{NaOH}$ and 3\% hydrogen peroxide respectively. The solution was incubated for $24 \mathrm{hr}$, neutralized and was analyzed in the developed method. The standard drug PBD was exposed to Ultraviolet light at $264 \mathrm{~nm}$ for $24 \mathrm{hr}$ for photolytic degradation and heated in a hot air oven at $60^{\circ} \mathrm{C}$ for $24 \mathrm{hr}$ for thermal degradation study. The peak area response of PBD in the stress degradation study was compared with the calibration curve results and the percentage of degradation was calculated. All samples diluted to expected concentration filtered through a $0.45 \mu \mathrm{m}$ filter and shifted into the autosampler vials prior to LC-MS analysis.

\section{RESULTS}

The LC-MS method for the analysis of PBD and characterization of degradation products were carried as per ICH guidelines. ${ }^{14-16}$ The systematic method optimization was completed after achieving the symmetric peak shape with an excellent resolution at the mobile phase composition of $\mathrm{pH} 6.4$ phosphate buffer and Methanol, Acetonitrile in the ratio of $45: 15: 40(\mathrm{v} / \mathrm{v})$. The flow rate of the mobile phase is $0.9 \mathrm{ml} /$ $\mathrm{min}$ in isocratic elution. UV detection was carried at a wavelength of 264 $\mathrm{nm}$ and separation was achieved on Prontosil ODS $\mathrm{C}_{18}$ Column (250X $4.6 \mathrm{~mm}, 5 \mu$ ). In the optimized conditions, well retained, resolved and symmetric peaks are observed in the standard chromatogram contains $100 \mu \mathrm{g} / \mathrm{mL}$ of PBD. The standard chromatogram of PBD obtained with optimized conditions was given in Figure 2.

The system suitability parameters were studied in the optimized method for PBD and the number of theoretical plates was found in 7854 and the tailing factor found 0.89 . The values confirm that all the system suitable parameters for PBD were found to be within the acceptable limits and hence the method was found to be suitable for the analysis of PBD.

Linear calibration curve observed within the concentration range of $0.5-250 \mu \mathrm{g} / \mathrm{mL}$ for PBD in the developed method. The linear regression equation was found to be $\mathrm{y}=3001 . \mathrm{x}+9962\left[R^{2}=0.999\right] \mathrm{PBD}$. Accurate

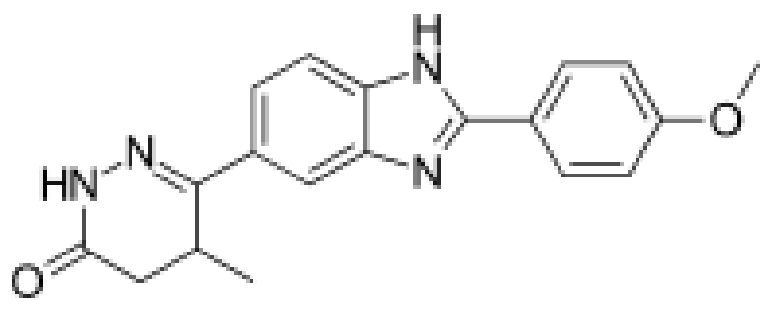

Figure 1: Chemical structure of Pimobendan. 
fit correlation coefficient value (more than 0.999) was observed for PBD confirms that the method follows linear relation accurately within the concentration range studied. Table 1 shows the linear calibration curve results of PBD in the developed method.

The \% RSD of the peak area responses observed in the precision (intra and interday) and ruggedness study was calculated and was found to be $0.96,0.81$ and 1.53 for PBD in intraday, interday precision and ruggedness study. The results confirm that the proposed method for PBD was found rugged and precise.

The robustness of the proposed method for the PBD was carried by a change in the composition of a mobile phase ratio, the flow rate of the mobile phase, a wavelength of the UV detector and column temperature. In all these changed conditions, the $\%$ change in peak area response was calculated and was found within the acceptable limit of less than 2 (Table 2) for the drug PBD confirms that the method was found to be robust.

Spiked recovery at $50 \%, 100 \%$ and $150 \%$ spiked levels of the standard at a $100 \%$ spiked level of $100 \mathrm{PBD}$ was studied. The result of the recovery study was given in Table 3 confirms that the method was found accurate.

\section{MS analysis of PBD}

Mass spectrometry analysis of $\mathrm{PBD}$ drug fragments was performed by introducing column eluting into equipment for Q1 and MS scan. Complete mass spectra were achieved in -ve ion and + ion modes. In the electrospray ionization spectra of $\mathrm{PBD}[\mathrm{M}+\mathrm{H}]$, positive ions were observed at an $\mathrm{m} / z$ value of 335.42 (Figure 3 ). The product-ions of 335.42 were observed at $\mathrm{m} / \mathrm{z}$ value of $319.18,287.19,277.09,261.08,250.16$, 223.30, 111.47 and 86.05 (Figure 3). Based on the experimental results,

Table 1: Results of Calibration test of PBD.

\begin{tabular}{ccc}
\hline S No & Concentration in $\mu \mathrm{g} / \mathrm{mL}$ & Peak Area \\
\hline 1 & 0.5 & 14253.2 \\
2 & 1 & 21938.7 \\
3 & 10 & 35983.5 \\
4 & 25 & 74758.2 \\
5 & 50 & 154716.3 \\
6 & 100 & 310296.5 \\
7 & 150 & 472198.2 \\
8 & 200 & 613624.7 \\
9 & 250 & 752583.6 \\
\hline
\end{tabular}

Table 2: Results of Robustness study.

\begin{tabular}{|c|c|c|c|c|}
\hline \multirow{2}{*}{$\begin{array}{l}S \\
\text { No }\end{array}$} & \multirow{2}{*}{$\begin{array}{l}\text { Changed } \\
\text { Condition }\end{array}$} & \multirow{2}{*}{ Condition studied } & \multicolumn{2}{|c|}{ PBD } \\
\hline & & & Peak Area & $\%$ Change \\
\hline 1 & Standard & Optimised & 310296.5 & --- \\
\hline 2 & MP 1 & $\begin{array}{c}\text { Methanol, Acetonitrile and } \\
\text { 0.1 phosphate buffer 40:20:40 } \\
(\mathrm{v} / \mathrm{v})\end{array}$ & 311316.1 & -0.33 \\
\hline 3 & MP 2 & $\begin{array}{l}\text { Methanol, Acetonitrile } \\
\text { and 0.1 phosphate buffer } \\
\text { 4050:10:40 (v/v) }\end{array}$ & 308367.8 & 0.62 \\
\hline 4 & PH & 6.3 & 305829.0 & 1.44 \\
\hline 5 & PH & 6.5 & 304637.9 & 1.82 \\
\hline 6 & WL 1 & $269 \mathrm{~nm}$ & 315756.1 & -1.76 \\
\hline 7 & WL 2 & $259 \mathrm{~nm}$ & 305186.5 & 1.65 \\
\hline
\end{tabular}

the reproducible mass fragmentation pattern was observed for PBD in the developed method.

\section{Forced degradation study}

Forced degradation study of PBD drug was performed under ICH guidelines on stability testing of new drug substances and products. The test was performed to study the effect of acidic, basic, peroxide, thermal and UV degradation. Stress degraded compounds of PBD drugs under various conditions were eluted into the mass spectrometer in order to identify the degradation products. The most rapid degradation was achieved under acidic and ultraviolet conditions. The acidic and ultraviolet conditions were found sensitive to PBD, were a total of four compounds, including three degraded products were identified in the chromatogram. After incubation, almost $10 \%$ of the drug was degraded and found $90 \%$. In acidic degradation (Figure $4 \mathrm{a}$ ), a total of three degradation compounds were detected along with PBD. The related compound $\mathrm{B}$ of $\mathrm{PBD}$ with a molecular weight of 352 was observed at a retention time of $8.5 \mathrm{~min}$ due to the breakage of $\mathrm{C}=\mathrm{N}$ bond in the structure with results in the formation of related compound $\mathrm{B}(\mathrm{m} / \mathrm{z}$ 353.25). The other two degradation products were observed at a retention time of 4.5 and $6.1 \mathrm{~min}$, corresponding to the $\mathrm{m} / z$ value of 287.19 and 250.16. In base degradation study (Figure 4b), additional degradation compounds correspond to related compound A of PBD at a retention time of $1.5 \mathrm{~min}$ with $\mathrm{m} / z$ value of 339.41 . A similar compound with the same $\mathrm{m} / \mathrm{z}$ values was observed for degradation products observed at retention tie of $1.6 \mathrm{~min}$ in peroxide degradation study (Figure 4c). This confirms that a similar type of bond breakage was observed in the peroxide degradation also. Very less \% degradation with two degradation products was observed in thermal degradation

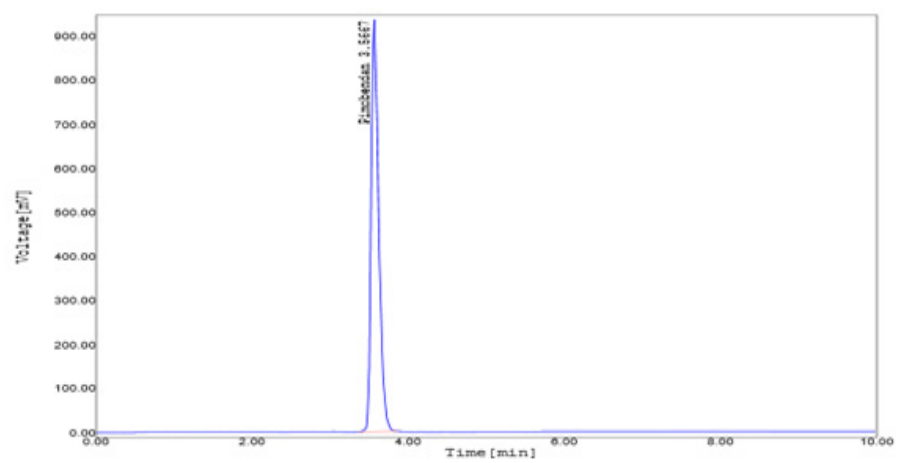

Figure 2: Standard chromatogram of PBD.

Table 3: Results of Recovery study.

\begin{tabular}{ccccccc}
\hline S No & Level & \multicolumn{4}{c}{ Concentration in $\mu \mathrm{g} / \mathrm{mL}$} & $\begin{array}{c}\% \text { of } \\
\text { recovery }\end{array}$ \\
\cline { 3 - 5 } & & Target & Spiked & Total & Amount received & \\
\hline 1 & & 100 & 50 & 150 & 147.46 & 98.30 \\
2 & $50 \%$ & 100 & 50 & 150 & 147.26 & 98.17 \\
3 & & 100 & 50 & 150 & 148.73 & 99.15 \\
4 & & 100 & 100 & 200 & 198.00 & 99.00 \\
5 & $100 \%$ & 100 & 100 & 200 & 197.05 & 98.53 \\
6 & & 100 & 100 & 200 & 197.16 & 98.58 \\
7 & & 100 & 150 & 250 & 248.93 & 99.57 \\
8 & $150 \%$ & 100 & 150 & 250 & 248.43 & 99.37 \\
9 & & 100 & 150 & 250 & 247.89 & 99.16 \\
\hline
\end{tabular}



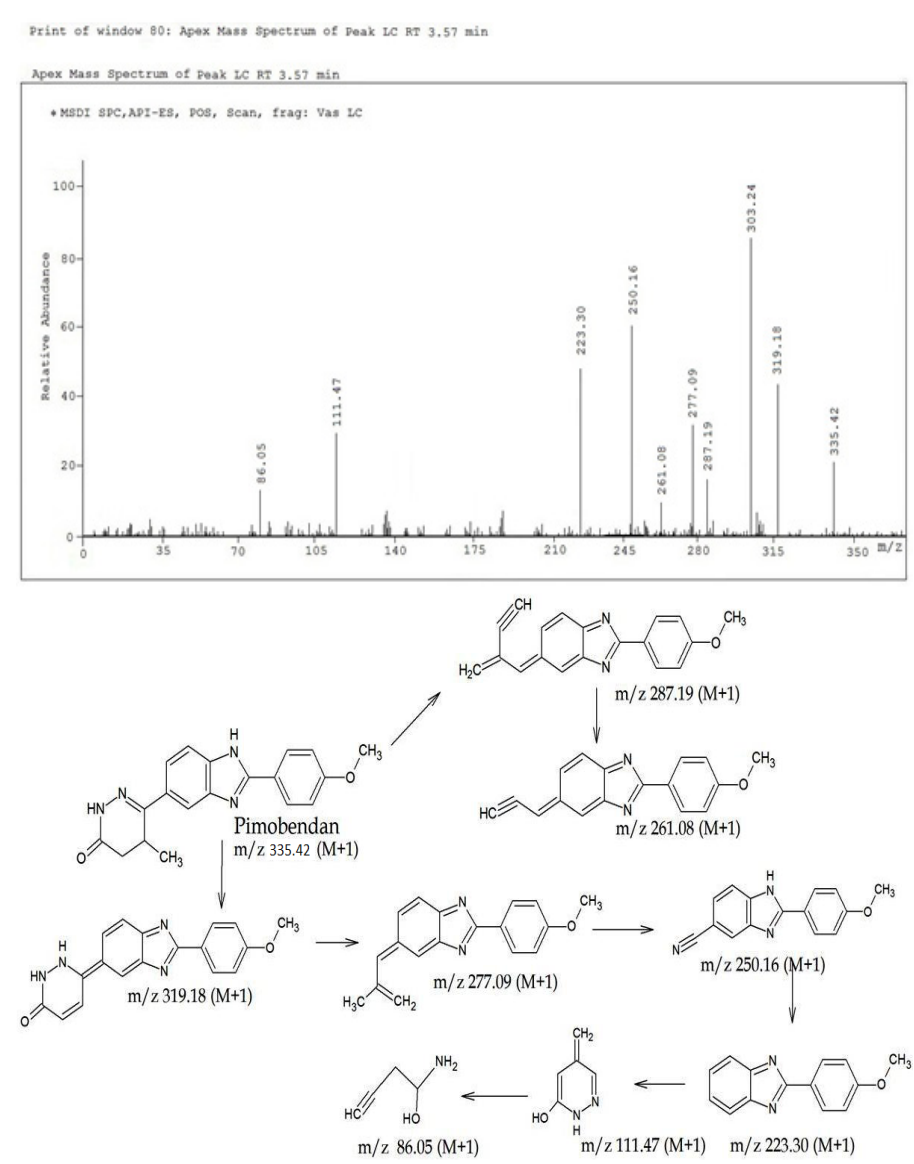

Figure 3: Parent and product ion mass spectrum of PBD and Proposed fragmentation pathways.

conditions (Figure 4d). In UV light degradation (Figure 4e), the high $\%$ degradation and three degradation products were observed. In this degradation study, the related compound B of PBD was observed. In all the stress conditions, the $\%$ degradation was found to be less than $2 \%$ and all the degradation conditions, the additional degradation compounds were effectively separated and precise mass spectra were observed in all the degradation compounds.

\section{DISCUSSION}

The present work aims to develop a simple, precise, accurate stabilityindicating LC-MS method for quantification of PBD, including degradation compounds. The maximum absorption wavelength $\left(\lambda_{\max }\right)$ of PBD was confirmed by scanning the standard drug in the wavelength range $200-400 \mathrm{~nm}$ in a UV spectrophotometer and confirms that at a wavelength of $264 \mathrm{~nm}$ was found to be suitable for the detection of PBD. In the method optimization study, system suitability, peak symmetry and responses factor are the key factors that are kept in consideration. The mobile phase was confirmed by the change in different solvents in different ratios, the strength of organic modifier and different strengths of $\mathrm{pH}$ modifier and confirms that $\mathrm{pH} 6.4$ phosphate buffer, methanol and acetonitrile in the ratio of 45:15:40 (v/v) at a flow rate of 0.9 $\mathrm{ml} / \mathrm{min}$ in isocratic elution was found to be suitable and the optimized separation was achieved on Prontosil ODS C ${ }_{18}$ Column (250X $4.6 \mathrm{~mm}, 5 \mu)$.

In the optimized conditions, the PBD elutes at a retention time of $3.5 \mathrm{~min}$ with acceptable system suitability of 7854 number of theoretical plates
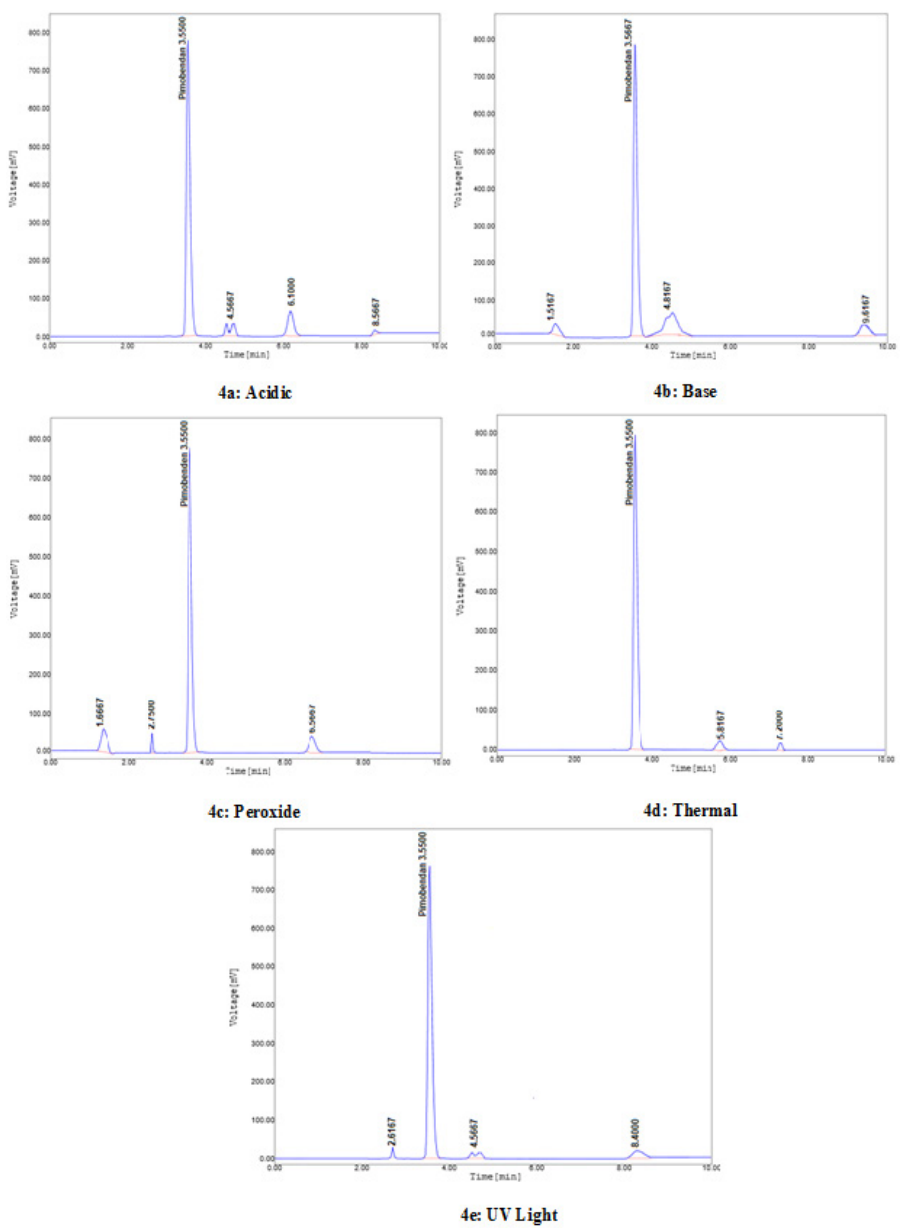

Figure 4: Forced Degradation Chromatograms of PBD in different stress degradation study.

and 0.89 tail factor with very high response factor. Hence the method was found to be suitable for the analysis of PBD.

The calibration curve was found to be linear within the concentration range of $0.5-250 \mu \mathrm{g} / \mathrm{mL}$ with regression equation of $\mathrm{y}=3001 . \mathrm{x}+$ $9962\left[R^{2}=0.999\right]$ and confirms that the method follows linear relation accurately within the concentration range studied.

The $\%$ RSD of the peak area responses observed in the precision (intra and interday) and ruggedness study was calculated and was found to be within the acceptable limits confirm that the proposed method for PBD was found rugged and precise. There is no considerable change in the peak area was observed when analysis was carried with small change in the developed method conditions in robustness study, this confirms that the method is found to be robust.

In the electrospray ionization spectra of $\mathrm{PBD}[\mathrm{M}+\mathrm{H}]$, positive ions were observed at an $\mathrm{m} / z$ value of 335.42 . The product-ions of 335.42 were observed at $\mathrm{m} / z$ value of $319.18,287.19,277.09,261.08,250.16,223.30$, 111.47 and 86.05. Based on the mass fragments observed in the MS spectra of $\mathrm{PBD}$, the fragmentation pattern of the PBD was evaluated.

The Forced degradation study of PBD was performed to study the effect of acidic, basic, peroxide, thermal and UV light on the stability and degradation of PBD in the corresponding stress effect. Stress degraded compounds of PBD drugs under various conditions were eluted into the mass spectrometer in order to identify the degradation products. The most rapid degradation was achieved under acidic and ultraviolet conditions. The acidic and ultraviolet conditions were found sensitive to 
PBD, were a total of four compounds, including three degraded products were identified in the chromatogram. In base degradation study, additional degradation compounds correspond to related compound $\mathrm{A}$ of PBD at a retention time of $1.5 \mathrm{~min}$ with $\mathrm{m} / z$ value of 339.41. A similar compound with the same $\mathrm{m} / \mathrm{z}$ values was observed for degradation products observed at retention time of $1.6 \mathrm{~min}$ in peroxide degradation study. This confirms that a similar type of bond breakage was observed in the peroxide degradation also. In all the degradation studies studied, the method can effectively separate the degradation compounds along with standard PBD and the degradation compounds were effectively characterized using mass spectra. In the mass spectra of degradation compounds and standard PBD in all the stress studies shows clear mass without any impurities confirms that the method can effectively separate the degradation compounds and the degradation compounds were characterized using mass spectra.

The earlier reported method reported by Makoto Asakura et al. ${ }^{9}$ is a simultaneous determination of the enantiomers of the pimobendan in rat plasma by HPLC. The other LC method reported by Twinkal D dobariyal $(2013)^{10}$ reported the method for estimation of pimobendan in pharmaceutical dosage forms. Though these reported methods are simple and economic than the present described, the application and sensitivity and selectivity with usage of LC-MS data is way advanced provides numerous information of the pimobendan drug and its derivates. Hence the present method is novel and applicable to study the pimodendan and its degradative products.

\section{CONCLUSION}

The quick and straightforward LC-MS strategy was developed for the investigation of PBD degradation in different stress conditions and the identification of the degradation products. The proposed LC-MS method for validated according to ICH Q2 (R1) guidelines. The proposed method was found accurate, precise and robust that can separate and identify the degradation products formed in stress conditions. The forced degradation response of the PBD drug was evaluated by exposing the drug to different stress conditions like acidic and base hydrolysis, oxidative, thermal and photolytic condition as per guidelines. The results of the proposed method shown that the PBD drug was stable under acidic and UV conditions. The proposed LC-MS method was found high specificity as it was able to separate the PBD drug as well as its degradation products.

\section{CONFLICT OF INTEREST}

The authors declare no conflict of interest.

\section{ABBREVIATIONS}

PBD: Pimobendan; RP-HPLC: Reverse Phase-High Performance Liquid Chromatography; LC-MS: Liquid Chromatography-Mass Spectrometry; ICH: International Conference on Harmonization; LOD: Limit of detection; LOQ: Limit of Quantification; RSD: Relative standard deviation.

\section{REFERENCES}

1. Galatulas I., Bossa R., Efstathiu G., and Ninci MA. Esorubicin cardiotoxicity in vitro: antagonism by pimobendane. Acta Physiol Hung. 1990;75:123-4.

2. Hanzlicek AS, Gehring R, Kukanich B, Kukanich KS, Borgarelli M, Smee N, et al. Pharmacokinetics of oral pimobendan in healthy cats. J Vet Cardiol. 2012;14(4):489-96

3. Gordon SG, Miller MW, and Saunders AB. Pimobendan in heart failure therapy-a silver bullet. J Am Anim Hosp Assoc. 2006;42(2):90-3.

4. Haggstrom J, Boswood A, Grady M. Effect of Pimobendan or Benazepril Hydrochloride on Survival Times in Dogs with Congestive Heart Failure Caused by Naturally Occurring Myxomatous Mitral Valve Disease: The QUEST Study. J. Vet. Intern. Med. 2008;22(5):1124-35.

5. Verdouw PD, Hartog JM, Duncker DJ, Roth W, and Saxena PR. Cardiovascular profile of pimobendan, a benzimidazole-pyridazinone derivative with vasodilating and inotropic properties. Eur J Pharmacol. 1986;126(1-2):21-30.

6. Peters P, Saborowski F, and Kothe A. Effect of pimobendan on peripheral hemodynamics. Z Kardiol. 1989;78(8):538-44.

7. Szepesi G, Gazdag M, Mihályfi K. Selection of high-performance liquid chromatographic methods in pharmaceutical analysis. III Method validation. J Chromatogr. 1989;464:265-78.

8. Carr GP, Wahlich JC. A practical approach to method validation in pharmaceutical analysis. J Pharm Biomed Anal. 1990;8(8-12):613-8.

9. Asakura M., Nagakura A., Tarui S., and Matsumura R. Simultaneous determination of the enantiomers of pimobendan and its main metabolite in rat plasma by high-performance liquid chromatography. J Chromatogr. 1993;614(1):135-41.

10. Twinkal, D, Dobariya, Parmeshwari J, and Multani. Development and Validation of Methods for Estimation of Pimobendan In Pharmaceutical Dosage Form. Int J Chemtech Res. 2013;5(5);2154-64.

11. $\mathrm{Wu} \mathrm{H}$, Guo J, and Chen $\mathrm{S}$. Recent developments in qualitative and quantitative analysis of phytochemical constituents and their metabolites using liquid chromatography-mass spectrometry. J Pharm Biomed Anal. 2013;72:267-91.

12. Chaimbault. and Patrick. The Modern Art of Identification of Natural Substances in Whole Plants. Recent Advances in Redox Active Plant and Microbial Products. Springer Netherlands. 2014:31-94.

13. Dass and Chhabil. Hyphenated Separation Techniques. Fundamentals of Contemporary Mass Spectrometry. John Wiley and Sons Inc. 2017:151-94.

14. International Conference of Harmonization. ICH, Q1A (R2), Stability testing of new drug substances and products. Geneva: International Conference on Harmonization. 2003

15. International Conference of Harmonization. ICH, Q1B, Stability testing: photostability testing of new drug substances and products. Geneva: International Conference on Harmonization. 1996

16. International Conference of Harmonization. $\mathrm{ICH}, \mathrm{Q} 2(\mathrm{R} 1)$, validation of analytical procedures: text and methodology. Geneva: International Conference on Harmonization. 1994. 